\title{
Agile DMAIC: Um Método para Avaliar e Melhorar o Uso do Scrum em Projetos de Software
}

\author{
Thiago Ferraz V. da Cunha, Rossana M. C. Andrade* \\ ${ }^{1}$ Grupo de Redes de Computadores, Engenharia de Software e Sistemas (GREat) \\ Mestrado em Ciência da Computação (MDCC) \\ Universidade Federal do Ceará (UFC), Fortaleza - Ceará - Brasil \\ \{thiagoferraz, rossana\} egreat.ufc.br
}

\begin{abstract}
Scrum embraces continuous improvement and, naturally, tends to evolve its own implementation over time. However, it can be misused, delaying its benefits in productivity and quality. There should be then a periodic assessment on how its principles and practices are understood and addressed to avoid its misuse. Some maturity models allow measuring how agile practices have been adopted, however, these models are usually general what leads to more time to apply them in the project. Then, this article proposes the Agile DMAIC, a method that aims to identify and address issues in the use of Scrum, providing qualitative and quantitative measurements on how far its application is from the best possible implementation. The Agile DMAIC is applied in seven real projects for evaluation and their results are also discussed in this article.
\end{abstract}

Resumo. Scrum abrange a melhoria contínua e, naturalmente, tende a evoluir a sua própria implementação ao longo do tempo. No entanto, ele pode ser mal utilizado, atrasando seus benefícios de produtividade e qualidade. Para que isso não ocorra, é necessária a avaliação periódica de como seus princípios e práticas são compreendidos e implementados. Alguns modelos de maturidade permitem medir a forma de adoção de práticas ágeis, entretanto, esses modelos usualmente são generalistas e por isso muito extensos. Então, este artigo propõe o Agile DMAIC, um método que visa identificar e solucionar problemas no uso do Scrum, fornecendo medições qualitativas e quantitativas do quão distante a sua aplicação está de sua melhor implementação possível. Para avaliar o Agile DMAIC, ele é aplicado em sete projetos reais e seus resultados são também discutidos neste artigo.

\section{Introdução}

O Scrum é um processo iterativo e incremental utilizado na gestão e desenvolvimento de projetos, que define uma estrutura base a partir de fundamentos, princípios, valores e práticas, e inclui orientações para a sua utilização [Schwaber 1995][Beedle et al. 2000]. A essa estrutura base são adicionadas práticas de engenharia e gestão para a instanciação do Scrum conforme o contexto e a necessidade de cada projeto [Rubin 2012].

A implementação do Scrum, portanto, é considerada única por projeto e sua adequada utilização depende de fatores como a experiência do time e do cliente, as competências desse time, a natureza do projeto, entre outros [Cohn 2009]. Esses fatores influenciam a forma na qual o Scrum deve ser instanciado para que seus benefícios sejam alcançados. Não é raro, todavia, casos em que o time do projeto sugere não mais utilizar o Scrum, devido a perdas de qualidade e produtividade durante a implantação ou nas primeiras iterações

\footnotetext{
*Bolsista de produtividade DT 2 (CNPq): 314021/2009-4
} 
[Moe and Dingsøyr 2008][Balkanski 2008]. Consequentemente, em alguns desses casos, a culpa de tais resultados é associada a nova metodologia adotada e não ao mau uso da mesma [Crescêncio 2013]. Nesse cenário, o time comumente volta a utilizar a abordagem anterior e uma segunda tentativa de adoção do Scrum se torna improvável.

O sucesso da implantação e continuidade com o Scrum depende, portanto, de como cada projeto instancia essa metodologia e, além disso, de como essa instanciação é ajustada conforme o projeto evolui. Existem estratégias de adoção do Scrum para que os resultados iniciais sejam mais assertivos, por exemplo, selecionar um projeto piloto com uma equipe pequena, que possua um patrocinador engajado e cuja importância seja a necessária para motivar os envolvidos [Cohn 2009]. Entretanto, mesmo após uma implantação bem sucedida, os resultados do projeto podem evoluir lentamente ou até mesmo retroceder.

Nesse contexto, surge a necessidade de gerenciar a implementação do Scrum. Para a boa utilização de práticas ágeis, existem modelos de maturidade e checklists que possibilitam medir a forma de adoção dessas práticas e, se aplicados corretamente, servem como guia de como melhorar a agilidade nos projetos [Qumer et al. 2007][Sidky et al. 2007]. Entretanto, por serem usualmente generalistas, esses modelos são muito extensos, focando em práticas de várias metodologias ao mesmo tempo, o que dificulta quando o projeto já se utiliza do Scrum e deseja inicialmente melhorar o uso dessa metodologia. Além disso, para a avaliação do Scrum, há a necessidade de checklists mais específicos, que forneçam uma visão de forças e fraquezas no uso dos princípios e práticas ágeis do Scrum.

Este trabalho propõe então um método sistemático para avaliação e melhoria do uso do Scrum em projetos de software. Esse método é chamado Agile DMAIC e pode ser utilizado tanto na adoção do Scrum quanto na melhoria de sua implementação em projetos que já o utilizam há várias iterações. O Agile DMAIC é baseado em princípios e técnicas de gestão da qualidade e visa aumentar o alinhamento da gestão de projeto aos princípios ágeis, com foco na melhoria do uso de princípios e práticas do Scrum.

Para apresentar o Agile DMAIC, este artigo está estruturado em mais cinco seções. A Seção 2 introduz o Scrum, Six Sigma e Lean Six Sigma. Os trabalhos relacionados ao uso de modelos de maturidade e checklists para avaliação de práticas ágeis são discutidos na Seção 3. O Agile DMAIC é proposto na Seção 4 e os resultados de sua aplicação em sete projetos reais são discutidos na Seção 5. Finalmente, na Seção 6, o artigo é concluído com as principais contribuições e os trabalhos futuros.

\section{Fundamentação Teórica}

A adoção de práticas ágeis no desenvolvimento de software tem se tornado uma resposta a necessidade de se produzir mais rápido, um software com melhor qualidade e que satisfaça as necessidades do usuário. A comunidade ágil reconhece as vantagens do uso dessas práticas e o Scrum representa uma alternativa frente as metodologias tradicionais de desenvolvimento. Já a adoção do Six Sigma e Lean Six Sigma vem crescendo em organizações de Tecnologia da Informação, onde são utilizados na melhoria de processos de desenvolvimento, colaborando na realização dos objetivos de negócio dessas organizações [Pan et al. 2007]. Essas metodologias são detalhadas nas próximas Subseções.

\subsection{Scrum}

O Scrum é uma metodologia ágil para gestão de projetos, desenvolvimento e manutenção de produtos de software, que adota uma abordagem empírica e pode ser utilizado em 
projetos complexos com, por exemplo, grande quantidade de produtos diferentes, uso simultâneo de várias tecnologias, elevado número de stakeholders, necessidade de conhecimentos técnicos especializados, muitas restrições tecnológicas e incertezas associadas [Schwaber 2007b][Schwaber 2007a]. O Scrum se baseia nos seguintes fundamentos:

- Transparência - todos os artefatos do projeto precisam estar visíveis aos envolvidos e as decisões e definições do projeto devem ser compartilhadas de forma efetiva;

- Inspeção - os artefatos são continuamente inspecionados e os problemas encontrados devem ser resolvidos o mais rápido possível; e

- Adaptação - a melhoria contínua deve ser executada em todas as frentes do projeto, com foco na melhoria da produtividade do time de desenvolvimento e da qualidade do produto.

O Scrum identifica três papéis: o Product Owner, que representa o cliente e é responsável pela gestão do escopo; o Scrum Master, que é o líder-servidor do time e responsável por garantir que o Scrum seja compreendido e seguido, e o Time de Desenvolvimento, que realiza o desenvolvimento propriamente dito. Os principais artefatos e eventos do Scrum são descritos a seguir:

- Product Backlog - conjunto completo dos requisitos conhecidos do produto, com os itens priorizados pelo Product Owner;

- Sprint - janela de tempo que corresponde a uma iteração;

- Sprint Backlog - contém as atividades planejadas pelo Time de Desenvolvimento para atender aos itens priorizados do Product Backlog;

- Sprint Planning - reunião de planejamento da Sprint realizada pelo Time de Desenvolvimento para a execução da Sprint;

- Daily Scrum - reunião envolvendo o time e o Scrum Master onde cada participante informa o que fez, o que fará e quais são os impedimentos que estão atrapalhando a execução das atividades;

- Revisão da Sprint - reunião em que o Product Owner revisa os softwares ou produtos entregues e aprova ou não a Sprint; e

- Retrospectiva da Sprint - reunião em que o time reporta os pontos positivos e negativos da Sprint passada, identificando as ações necessárias para que os problemas encontrados sejam evitados nas Sprints futuras.

Embora existam muitas informações e treinamentos sobre o Scrum, a adoção dessa metodologia é particular ao projeto e à organização e, dessa forma, cada implantação tornase um desafio único e que não deve ser subestimado. Surge, portanto, a necessidade de gerenciar adequadamente essa adoção de acordo com as necessidades de cada projeto, bem como gerenciar a utilização do Scrum a médio e longo prazo.

\subsection{Six Sigma e Lean Six Sigma}

O Six Sigma é uma metodologia quantitativa que objetiva aumentar o desempenho e a lucratividade das empresas. Criado em meados de 1980, o Six Sigma é amplamente utilizado por seus resultados comprovados e é considerado como "a metodologia de qualidade do século XXI" [Werkema 2012]. Essa metodologia melhora os resultados financeiros e de qualidade das empresas a partir da identificação e remoção das causas de defeitos e da diminuição da variabilidade de produtos, processos e serviços.

O Six Sigma utiliza um conjunto de métodos e ferramentas de gestão de qualidade, incluindo métodos estatísticos, para realizar a análise detalhada dos processos, a fim de descobrir as causas dos defeitos. Isso é conseguido através do uso de duas submetodologias, 
o DMAIC e DMADV. O primeiro é utilizado para a melhoria de processos existentes, enquanto o segundo é utilizado para o desenvolvimento de novos processos ou produtos em níveis de qualidade Six Sigma. As fases dessas submetodologias são sumarizadas na Tabela 1 [George 2003]. O Six Sigma cria uma infraestrutura de pessoas que são especialistas nesses métodos. Cada projeto Six Sigma segue uma sequência definida de passos e tem metas quantificadas de redução de custos financeiros (e aumento do lucro).

Tabela 1. Sub-metodologias DMAIC e DMADV, adaptado de [George 2003].

\begin{tabular}{|c|c|c|}
\hline & Fase & Descrição \\
\hline \multirow{5}{*}{ 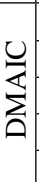 } & Definir (Define) & Definir os objetivos do projeto e de clientes (interno e externo) \\
\hline & Medir (Measure) & Medir o processo para determinar o desempenho atual \\
\hline & Analisar (Analyze) & Analisar e determinar as causas de defeitos \\
\hline & Melhorar (Improve) & Melhorar o processo a partir da eliminação de defeitos \\
\hline & Controlar (Control) & Controlar o desempenho do processo após implantação de melhorias \\
\hline \multirow{5}{*}{ 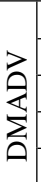 } & Definir (Define) & Definir os objetivos do projeto e de clientes (interno e externo) \\
\hline & Medir (Measure) & Determinar as especificações do cliente \\
\hline & Analisar (Analyze) & Analisar o processo quanto ao atendimento das necessidades do cliente \\
\hline & Projetar (Design) & Realizar o projeto do processo com foco nas necessidades do cliente \\
\hline & Verificar (Verify) & Verificar se o desempenho e a capacidade do novo processo atendem às necessidades do cliente \\
\hline
\end{tabular}

O Lean Six Sigma (LSS) é uma metodologia para definição e melhoria de produtos, processos e serviços com foco na redução de defeitos ou falhas, na eliminação da variação e dos desperdícios, priorizando, de forma planejada e objetiva, a obtenção de resultados de qualidade e financeiros [George 2003]. Essa metodologia consiste na integração dos princípios e técnicas do Lean [Poppendieck 2005] e do Six Sigma e potencializa os benefícios dessas duas metodologias, aliando os ganhos em velocidade do Lean aos ganhos em qualidade do Six Sigma.

O LSS identifica os seguintes papéis: Liderança Executiva, que é a alta gerência responsável pela visão estratégica da organização, a qual será utilizada para a implantação e execução do LSS; Champions, que assumem a responsabilidade pela implantação da metodologia na organização; Master Black Belts, que atuam como orientadores internos; Black Belts, que aplicam a metodologia em projetos específicos e Green Belts, que se ocupam da execução dos projetos $L S S$, juntamente com suas outras responsabilidades, sob a orientação de Black Belts.

\section{Trabalhos Relacionados}

Nesta Seção são analisados os trabalhos relacionados à adoção, melhoria e avaliação de práticas ágeis, modelos de maturidade e checklists, bem como sobre o uso do DMAIC em projetos de software.

Sidky et al. (2007) propõem o Agile Adoption Framework ( $A A F)$ como um processo para adoção de práticas ágeis. $\mathrm{O} A A F$ é composto de dois componentes, o Índice de Medição Ágil, que identifica o potencial ágil alcançável por projeto e organização; e o Processo de 4-Estágios, que auxilia a avaliar se o projeto e a organização estão aptos a avançar na adoção de práticas ágeis e a identificar quais práticas devem ser adotadas. Para a avaliação de agilidade são utilizados aproximadamente 300 indicadores, que são questões do tipo escala de classificação (uma única classificação é selecionada para a questão, entre uma sequência gradual de classificações possíveis). Esses indicadores são agrupados por público alvo (desenvolvedores, gerentes e avaliadores da qualidade) e, por observação e entrevistas, são classificados para medir o atendimento das características relacionadas aos princípios e 
práticas ágeis. Se por um lado, essa quantidade de indicadores torna o método abrangente, por outro lado, o torna extenso, demandando esforço significativo em sua utilização. Além disso, $\mathrm{O} A A F$ não se restringe a uma metodologia ágil específica e, no entanto, não há restrições em utilizá-lo, por exemplo, com o Scrum.

Vode e Sutherland (2008) propõem o Nokia Test para a avaliação do uso do Scrum em projetos de software. Esse checklist foi elaborado em 2005 e, incialmente, focava no desenvolvimento ágil. Em 2007, ele foi ajustado para avaliar as práticas do Scrum e, em 2008, Jeff Sutherland, cocriador do Scrum, estendeu o mesmo de forma a fornecer uma pontuação do uso do Scrum. O Nokia Test possui 50 itens e foca na avaliação do uso de artefatos e papéis do Scrum. Entretanto, ele é pouco abrangente e não avalia, por exemplo, parte dos princípios e valores, em relação aos eventos, avalia apenas a Sprint e, sobre os papéis, não há itens a respeito do Scrum Master.

Kniberg (2009) propõe o CRISP Scrum-Checklist como guia para os primeiros passos com o Scrum e para avaliar a implementação dessa metodologia nos projetos. Esse checklist foi elaborado em 2008 e tem como principal público-alvo as equipes iniciantes no Scrum. O CRISP Scrum-Checklist possui 78 itens e foca na avaliação de eventos e papéis. Esses itens são organizados por seções, cada uma associada implicitamente a uma prioridade. Ao contrário do Nokia Test, o CRISP Scrum-Checklist é bastante abrangente. Entretanto, por incluir a avaliação de práticas recomendadas mas não obrigatórias ele se torna desnecessariamente extenso.

Bezerra et al. (2007) propõem o MiniDMAIC como uma simplificação do DMAIC para tratar causas e resolução de problemas em projetos de software. Essa simplificação tem como objetivo reduzir a duração do DMAIC e, por conseguinte, o seu custo, associando-o ao tratamento de riscos dos projetos. O MiniDMAIC é uma simplificação do DMAIC voltada ao desenvolvimento de software, entretanto, seu foco é o tratamento de problemas gerais, como desvios na produtividade, prazo, densidade de projetos, entre outros.

Cunha et al. (2011) apresentam uma abordagem de integração do Scrum ao Lean Six Sigma (SLeSS). Essa abordagem de gestão de projetos e melhoria de processos é utilizada no desenvolvimento de customizações de software para celulares. O SLeSS define mecanismos para a integração dessas metodologias e um deles é a aplicação de princípios e técnicas do Lean Six Sigma para melhorar o uso do Scrum. Entretanto, a ausência de documentação e o uso do DMAIC sem simplificações torna esse mecanismo sobre a avaliação e melhoria do Scrum de difícil reuso e extenso.

O Agile DMAIC, método proposto neste trabalho, é resultado da continuação da pesquisa apresentada em [Cunha et al. 2011] e tem como objetivo aprimorar a abordagem SLeSS com foco na avaliação das práticas e princípios do Scrum. Em relação aos outros trabalhos apresentados, o Agile DMAIC pretende ser menos extenso, como o MiniDMAIC [Bezerra et al. 2007], mas sendo específico para avaliação e melhoria do Scrum. Vale ressaltar que ele pode ser utilizado em conjunto com o MiniDMAIC, mas possuem objetivos distintos.

\section{Agile DMAIC}

Nesta Seção apresentamos o Agile DMAIC, que é um método sistemático proposto neste trabalho para avaliar e melhorar o uso do Scrum em projetos de software. Esse método pode ser utilizado tanto na adoção do Scrum quanto na melhoria de sua implementação. Os objetivos do Agile DMAIC podem ser resumidos a seguir: 
$\checkmark$ Possibilitar a gestão quantitativa da implementação do Scrum;

$\checkmark$ Avaliar o uso de princípios e práticas do Scrum por projeto e a nível institucional; e

$\checkmark$ Identificar e tratar as causas de problemas para a evolução da implementação do Scrum, considerando o contexto e a necessidade dos projetos e clientes.

O Agile DMAIC utiliza princípios e técnicas do Lean Six Sigma e foca na execução, de forma iterativa e incremental, de uma versão simplificada do DMAIC [Tayntor 2007]. A execução do Agile DMAIC é realizada em paralelo à execução de Sprints do Scrum conforme apresentado na Figura 1. À medida que problemas no uso do Scrum são identificados e tratados, as melhorias são implantadas a cada Sprint do Agile DMAIC.

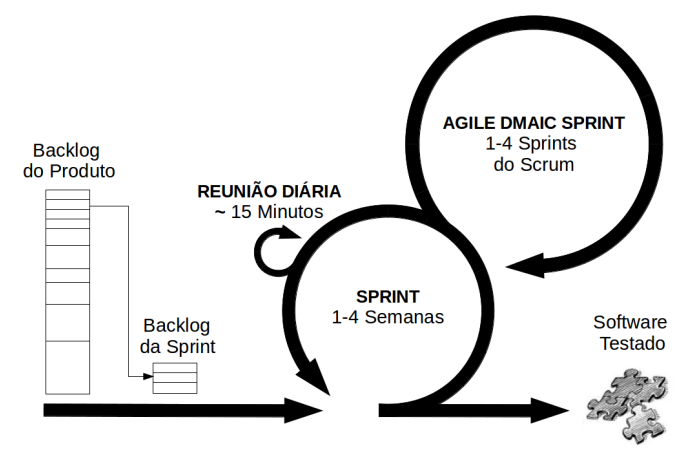

Figura 1. Execução Iterativa e Incremental do Agile DMAIC

Enquanto as Sprints do Scrum tem tamanho de 1 a 4 semanas [Schwaber and Sutherland 2011], as Sprints de execução do Agile DMAIC são de 1 a 4 vezes o tamanho de uma Sprint do Scrum. Esse tamanho foi assim definido com base nos experimentos realizados no estudo de caso deste trabalho. Por exemplo, em um projeto cuja Sprint do Scrum é de 2 semanas, uma Sprint do Agile DMAIC pode ser de 2 a 8 semanas, de forma que no máximo a cada 4 Sprints do Scrum, problemas no uso dessa metodologia sejam identificados, tratados e melhorias sejam implantadas com seus respectivos planos de controle. Também, por analogia, assim como o objetivo principal de uma Sprint do Scrum é a entrega de software testado, o objetivo de uma Sprint do Agile DMAIC é a implantação de melhorias no uso de princípios e práticas do Scrum.

Uma visão geral do Agile DMAIC é apresentada na Figura 2 e os detalhes são apresentados no decorrer desta Seção. O Agile DMAIC é composto de três componentes:

- Índice de Medição do Nível do Scrum (IMeNS), que avalia a adoção das práticas do Scrum por projeto e a nível institucional;

- Checklist de Avaliação do Scrum (CAS), utilizado para coletar a percepção das equipes quanto ao uso de princípios e práticas do Scrum; e

- DMAIC [Tayntor 2007], uma submetodologia do Lean Six Sigma [George 2003] voltada a melhoria de processos. No método proposto neste trabalho, o DMAIC é utilizado para a definição de metas, identificação e análise de causas raízes, implantação e controle de melhorias para possibilitar a gestão quantitativa da implementação do Scrum.

O Agile DMAIC se baseia em coletar periodicamente a percepção da equipe do projeto (Product Owner, Scrum Master e Time) em relação ao uso do Scrum e, a partir dessa percepção, identificar possíveis problemas em sua implementação. O resultado da aplicação do $C A S$ é utilizado como insumo na identificação de problemas e, além desse checklist, as 


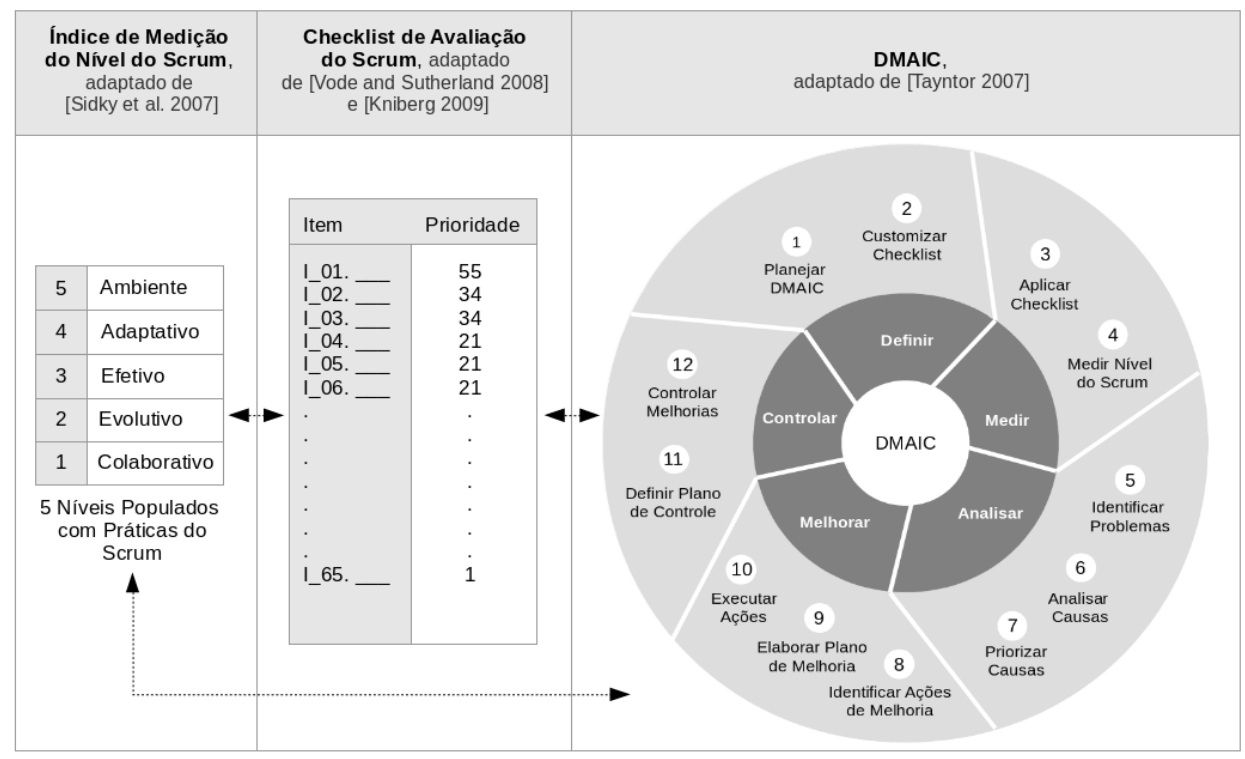

Figura 2. Visão Geral do Agile DMAIC

Reuniões de Revisão e Retrospectivas também podem ser fontes de identificação de problemas. A partir da aplicação do $C A S$ e da análise da coleta é possível classificar o nível do Scrum, utilizando o IMeNS, e identificar onde a equipe pode atuar para melhorar seus resultados com essa metodologia. Uma vez identificados os problemas, é necessário analisar as causas, definir e implantar ações de melhoria, antencipando os benefícios do Scrum, como a melhoria na produtividade do time e na qualidade do produto [Dybå and Dingsøyr 2008].

Dessa forma, o Agile DMAIC é utilizado como ferramenta para a definição de uma meta de melhoria no uso do Scrum, na análise de seu estado atual, na identificação e implantação de melhorias e no controle das mesmas para que o uso dessa metodologia continue em constante evolução.

\section{1. Índice de Medição do Nível do Scrum (IMeNS)}

O IMeNS é utilizado para classificar os projetos quanto ao nível de adoção de princípios e práticas do Scrum. Esse índice é uma especialização do Índice de Medição Ágil especificado no Agile Adoption Framework (AAF) [Sidky et al. 2007], a partir de sua simplificação ao remover as práticas e conceitos não relacionados ao Scrum. Como exemplo, o Test Driven Development [Beck 2002] e a Programação em Pares [Beck and Andres 2004] foram removidos do mapeamento proposto em [Sidky et al. 2007] pois, embora não haja restrições em usá-los com o Scrum, não são especificados como práticas dessa metodologia. Dessa forma, o Agile DMAIC adaptou apenas o Índice de Medição Ágil definido por Sidky et al. (2007) e não se utilizou, por exemplo, dos indicadores e demais componentes do $A A F$.

Os níveis definidos no IMeNS são, do 1 ao 5 (veja Figura 2), respectivamente: Colaborativo, promove a comunicação e colaboração entre os envolvidos do projeto; Evolutivo, foca na entrega contínua de software; Efetivo, objetiva o desenvolvimento de software testado e de alta qualidade, realizado de forma eficiente e efetiva; Adaptativo, estabelece as práticas que atuam para agilizar as respostas às mudanças; e Ambiente, que promove um ambiente altamente produtivo. Esses níveis são acessados a partir de práticas associadas a princípios ágeis do Scrum cujo mapeamento é apresentado na Tabela 2. Como exemplo, para acessar o nível 2 é necessário que o projeto atenda as práticas do nível 1 (P1 a P6) e 
do nível 2 (P7 a P9). Da mesma forma, para acessar ao nível 5 é necessário que o projeto atenda a todas as 17 práticas mapeadas nessa Tabela.

Tabela 2. Os 5 Níveis do IMeNS Populados com Práticas do Scrum, Adaptado de [Sidky et al. 2007]

\begin{tabular}{|c|c|c|c|c|c|}
\hline & \multicolumn{5}{|c|}{$\begin{array}{l}\text { Princípios Ágeis do Scrum } \\
\end{array}$} \\
\hline & $\begin{array}{l}\text { Abraçar mudanças } \\
\text { para entregar valor } \\
\text { ao cliente }\end{array}$ & $\begin{array}{l}\text { Planejar e } \\
\text { entregar software } \\
\text { com frequência }\end{array}$ & Focar nas pessoas & $\begin{array}{l}\text { Excelência } \\
\text { técnica }\end{array}$ & $\begin{array}{l}\text { Colaboração com } \\
\text { o cliente }\end{array}$ \\
\hline $\begin{array}{l}\text { Nível } 5 \\
\text { Ambiente }\end{array}$ & \begin{tabular}{|l|} 
[P16] Iterações \\
orientadas ao \\
cliente, [P17] \\
Feedbacks \\
contínuos de sua \\
satisfação
\end{tabular} & & & & \\
\hline $\begin{array}{l}\text { Nível } 4 \\
\text { Adaptativo }\end{array}$ & & $\begin{array}{l}\text { [P12] Entregas } \\
\text { menores e } \\
\text { frequentes, [P13] } \\
\text { Planejamento } \\
\text { adaptativo }\end{array}$ & & $\begin{array}{l}\text { [P14] Monitorar o } \\
\text { progresso diário }\end{array}$ & $\begin{array}{l}\text { [P15] Cliente } \\
\text { prontamente } \\
\text { acessível }\end{array}$ \\
\hline $\begin{array}{l}\text { Nível } 3 \\
\text { Efetivo }\end{array}$ & & & $\begin{array}{l}\text { [P10] Times } \\
\text { auto-organizáveis, } \\
\text { [P11] Comunicação } \\
\text { face a face }\end{array}$ & & \\
\hline $\begin{array}{l}\text { Nível } 2 \\
\text { Evolutivo }\end{array}$ & & $\begin{array}{l}\text { [P7] Entregas } \\
\text { contínuas, [P8] } \\
\text { Planejamentos em } \\
\text { níveis distintos } \\
\end{array}$ & & $\begin{array}{l}\text { [P9] Monitorar o } \\
\text { status da iteração }\end{array}$ & \\
\hline $\begin{array}{l}\text { Nível } 1 \\
\text { Colaborativo }\end{array}$ & $\begin{array}{l}\text { [P1] Reflexão e } \\
\text { ajuste do processo }\end{array}$ & $\begin{array}{l}\text { [P2] } \\
\text { Planejamento } \\
\text { colaborativo }\end{array}$ & $\begin{array}{l}\text { [P3] Equipes } \\
\text { colaborativas, [P4] } \\
\text { responsáveis e } \\
\text { motivadas }\end{array}$ & $\begin{array}{l}\text { [P5] Tarefas } \\
\text { executadas de } \\
\text { forma voluntária }\end{array}$ & $\begin{array}{l}\text { P6] } \\
\text { Compromisso do } \\
\text { cliente no } \\
\text { trabalho com a } \\
\text { equipe }\end{array}$ \\
\hline
\end{tabular}

\subsection{Checklist de Avaliação do Scrum (CAS)}

O CAS $S^{1}$ foi elaborado a partir do Nokia Test [Vode and Sutherland 2008] e do CRISP ScrumChecklist [Kniberg 2009] com o intuito de consolidar o melhor desses checklists e solucionar os pontos de melhoria dos mesmos. Esse novo checklist é aplicado à equipe do projeto (Product Owner, Scrum Master e o Time) para obter, a partir da percepção dessa equipe, as forças e fraquezas da atual implementação do Scrum.

O CAS é formado de 65 itens (I_01 a I_65) distríbuidos em treze seções, ordenadas de forma decrescente quanto a prioridade das mesmas em relação aos benefícios do Scrum. Cada item possui uma priorização ( 1 a 55, utilizando a sequência de Fibonacci, sendo 1 a menor e 55 a maior prioridade) e essa priorização foi predefinida com base na experimentação do Agile DMAIC nos projetos reais utilizados como estudo de caso neste trabalho e pode ser customizada pela equipe. A Figura 3 apresenta algumas seções e itens do CAS. Por exemplo, a seção Planejamento do Sprint possui 8 itens (I_04 a I_11). O primeiro item dessa seção verifica se há planejamento a cada iteração e possui prioridade 34 (P_34).

O CAS foca em avaliar como são utilizados os eventos e artefatos do Scrum. Entretanto também abrange bem os papéis, fundamentos, valores e princípios dessa metodologia. Para uma comparação entre os checklists analisados neste trabalho, os gráficos de abrangência de práticas do Scrum são apresentados na Figura 4 para o Nokia Test, CRISP Scrum-

\footnotetext{
${ }^{1}$ http://www.great.ufc.br/CAS/Checklist_de_Avaliacao_do_Scrum(CAS).pdf
} 

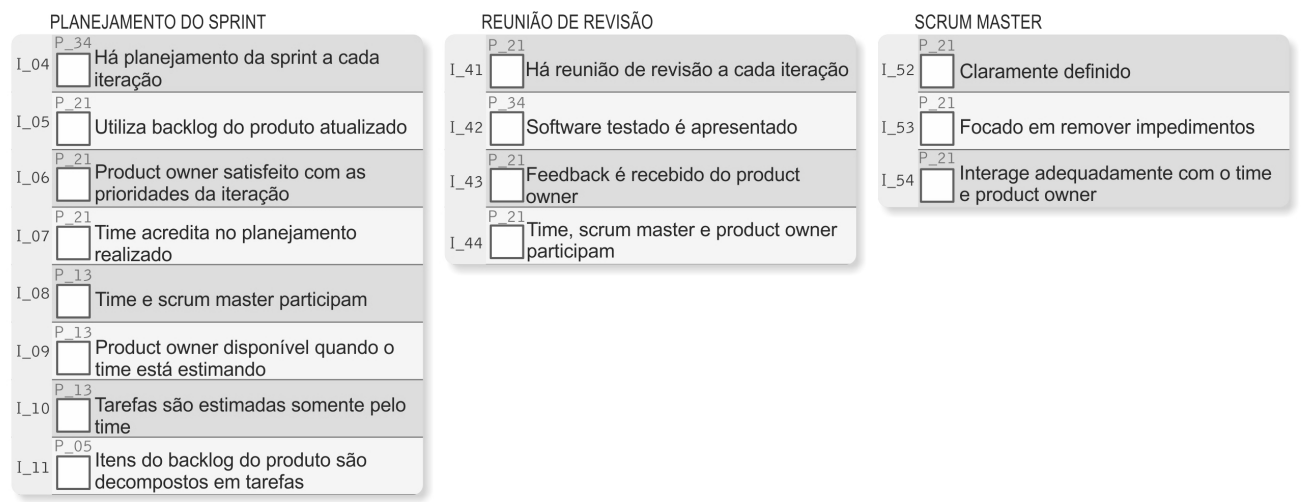

Figura 3. Algumas Seções e Itens do Checklist de Avaliação do Scrum (CAS)

Checklist e o CAS, respectivamente. O Nokia Test possui itens a respeito do Backlog do Produto, Sprint, Plano de Lançamentos, Gráfico de Burndown, Product Owner, Time, Fundamentos, Valores e Princípios. Entretanto, algumas práticas do Scrum não são abrangidas por esse checklist, como exemplo, não há itens sobre a Retrospectiva, Reunião de Revisão, Scrum Master, entre outros. Já o CRISP Scrum-Checklist não abrange diretamente apenas um dos fundamentos (inspeção) e o $C A S$ abrange todas as categorias identificadas.

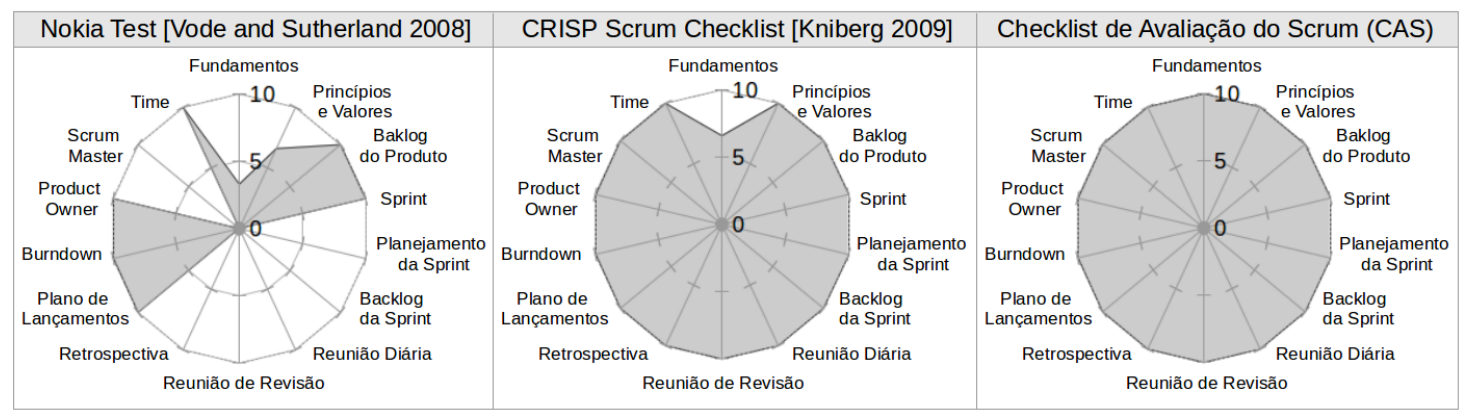

Figura 4. Abrangência dos Checklists Quanto às Práticas Ágeis do Scrum

A utilização do $C A S$, em conjunto com o IMeNS, possibilita classificar o nível do Scrum e serve como guia na adoção e melhoria do uso dessa metodologia. O atendimento das práticas do IMeNS é verificado a partir da aplicação do $C A S$ à equipe do projeto e do mapeamento de práticas do IMeNS aos itens do CAS, conforme apresentado na Tabela 3. Como exemplo, a prática Feedbacks contínuos da satisfação do cliente (P17) é verificada a partir do atendimento do projeto aos itens do CAS do I_38 ao I_44.

\subsection{DMAIC}

O Agile DMAIC propõe uma simplificação do DMAIC [Tayntor 2007] para a avaliação e melhoria do uso do Scrum em projetos de software. Nessa simplificação, o CAS é utilizado para a coleta da percepção da equipe e o nível do Scrum é calculado a partir dessa percepção e da priorização de itens do $C A S$, customizado pela equipe. As fases e passos do DMAIC utilizado pelo Agile DMAIC são apresentados na Tabela 4.

A customização dos itens e da priorização do $C A S$ é realizada de acordo com o contexto do projeto e cliente e visa identificar o máximo nível possível do Scrum para o projeto, isso porque podem existir restrições impostas pelo negócio, cliente, ou pela organização que 
Tabela 3. Mapeamento de Práticas do IMeNS aos Itens do CAS

\begin{tabular}{|c|c|}
\hline & Prática $<->$ Itens \\
\hline \multirow{2}{*}{$\begin{array}{l}\text { Nível } 5 \\
\text { Ambiente }\end{array}$} & P17 <->I_38 a I_44 \\
\hline & P16 <->I_02, I_05, I_06, I_32, I_33, I_49 \\
\hline \multirow{4}{*}{$\begin{array}{l}\text { Nível } 4 \\
\text { Adaptativo }\end{array}$} & P15 «> I_06, I_09, I_38 a I_40, I_43 \\
\hline & P14 $<>I$ I6, I_18, I_30, I_37, I_57, I_59 a I_65 \\
\hline & P13 $<>$ I_04, I_18, I_36, I_49, I_55 \\
\hline & P12 <> I_01, I_12, I_13, I_19, I_20, I_22, I_23 \\
\hline \multirow{2}{*}{$\begin{array}{l}\text { Nível } 3 \\
\text { Efetivo }\end{array}$} & P11 <-> I_08, I_09, I_40, I_41, I_43, I_44, I_48, I_54, 59, I_60, I_62 \\
\hline & P10 <-> I_25 a I_29, I_34, I_35, I_51 a I_54, I_56, I_58 \\
\hline \multirow{3}{*}{$\begin{array}{l}\text { Nível } 2 \\
\text { Evolutivo }\end{array}$} & P9 く-> I_16, I_18, I_24, I_30, I_37, I_63, I_65 \\
\hline & P8 $<->$ I_04, I_05, I_09, I_11, I_17, I_18, I_21, I_24, I_33, I_35, I_49, I_50, I_55 \\
\hline & P7 <-> I_01, I_13, I_19, I_20 \\
\hline \multirow{6}{*}{$\begin{array}{l}\text { Nível } 1 \\
\text { Colaborativo }\end{array}$} & P6 <-> I_09, I_38 a I_40 \\
\hline & P5 $<>$ I_04, I_07, I_08, I_10, I_11, I_17, I_24, I_35, I_56, I_58 \\
\hline & P4 <-> I_07, I_48, I_52 a I_54 \\
\hline & P3 <-> I_08, I_31, I_35, I_44, I_48, I_52 a I_54 \\
\hline & P2 <-> I_17, I_35, I_37, I_49 a I_51, I_55 a I_57 \\
\hline & P1 <-> I_03, I_14, I_15, I_41 a I_47, I_50 \\
\hline
\end{tabular}

Tabela 4. Fases e Passos do DMAIC Simplificado pelo Agile DMAIC

\begin{tabular}{|c|c|c|c|}
\hline \multirow{3}{*}{ อ } & \multicolumn{2}{|r|}{ Passo } & Descrição \\
\hline & 1 & Planejar DMAIC & $\begin{array}{l}\text { Elaborar plano de execução do } D M A I C \text {, especificando a meta de melhoria do nível do } \\
\text { Scrum por projeto e a nível institucional, o escopo de atuação (projetos alvo), o responsável } \\
\text { (Green Belt) pelo DMAIC e demais envolvidos }\end{array}$ \\
\hline & 2 & $\begin{array}{l}\text { Customizar } \\
\text { Checklist }\end{array}$ & $\begin{array}{l}\text { Definir a frequência de aplicação do } C A S \text { e customizá-lo (revisão de itens e priorização) de } \\
\text { acordo com o projeto e cliente. Por exemplo, por necessidade do negócio, o cliente define } \\
\text { que o software só seja implantado em seu ambiente ao final do projeto e, dessa forma, o } \\
\text { item I_13 do CAS deixa de ser prioridade }\end{array}$ \\
\hline & 3 & Aplicar Checklist & $\begin{array}{l}\text { O CAS é aplicado à equipe do projeto (Product Owner, Scrum Master e o Time). Seções do } \\
C A S \text { completamente atendidas podem ser excluídas em aplicações seguintes até que haja } \\
\text { indicativo de regressão }\end{array}$ \\
\hline 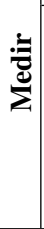 & 4 & $\begin{array}{l}\text { Medir Nível do } \\
\text { Scrum }\end{array}$ & $\begin{array}{l}\text { A coleta é validada e itens com menos de } 25 \% \text { e mais de } 75 \% \text { de pontuação, em relação ao } \\
\text { tamanho da equipe, são definidos como não atendidos e atendidos, respectivamente. Itens } \\
\text { fora desses intervalos precisam ser revisados em conjunto com a equipe. A classificação } \\
\text { do nível do } S c r u m \text { é acessada pelos mapeamentos das Tabelas } 2 \text { e } 3 \text {. O indicador do nível } \\
\text { do } S c r u m \text { é a razão da soma de priorizações de itens atendidos pela soma de todas as } \\
\text { priorizações, multiplicada por } 10 \text {, ou seja, } 10 * \frac{\sum P(\text { atendidos })}{\sum P}\end{array}$ \\
\hline \multirow{3}{*}{ 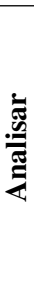 } & 5 & $\begin{array}{l}\text { Identificar } \\
\text { Problemas }\end{array}$ & $\begin{array}{l}\text { Os itens não atendidos pelo projeto são considerados problemas e são priorizados conforme } \\
\text { customizados pela equipe no } C A S\end{array}$ \\
\hline & 6 & Analisar Causas & $\begin{array}{l}\text { De posse da coleta e de problemas já identificados, é possível discernir entre causas rela- } \\
\text { cionadas ao entendimento da equipe, desconhecimento de princípios e práticas, falha na } \\
\text { comunicação ou demais causas, as quais podem ser identificadas com uso de técnicas do } \\
\text { Lean Six Sigma }\end{array}$ \\
\hline & 7 & Priorizar Causas & $\begin{array}{l}\text { As causas são priorizadas a partir das análises realizadas, da priorização do } C A S \text { e dos } \\
\text { mapeamentos de níveis e práticas das Tabelas } 2 \text { e } 3\end{array}$ \\
\hline \multirow{3}{*}{ | } & 8 & $\begin{array}{l}\text { Identificar Ações } \\
\text { de Melhoria }\end{array}$ & $\begin{array}{l}\text { Uma vez identificadas as causas raízes dos problemas, a definição de ações de melhoria é } \\
\text { realizada com a equipe }\end{array}$ \\
\hline & 9 & $\begin{array}{l}\text { Elaborar Plano de } \\
\text { Melhoria }\end{array}$ & $\begin{array}{l}\text { O plano de melhoria estabele a priorização das ações, os prazos e responsáveis pela execu- } \\
\text { ção e acompanhamento dessas ações }\end{array}$ \\
\hline & 10 & Executar Ações & As ações são executadas conforme estabelecido no plano de melhoria \\
\hline \multirow{2}{*}{ 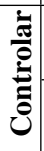 } & 11 & $\begin{array}{l}\text { Definir Plano de } \\
\text { Controle }\end{array}$ & $\begin{array}{l}\text { Avaliar riscos, definir gatilhos, ações, responsáveis e prazos, para assegurar que as melho- } \\
\text { rias já implantadas sejam duradouras }\end{array}$ \\
\hline & 12 & $\begin{array}{l}\text { Controlar } \\
\text { Melhorias }\end{array}$ & Controlar o projeto conforme definido no plano de controle \\
\hline
\end{tabular}


dificultem ou inviabilizem o uso de algumas práticas do Scrum. Essas alterações no DMAIC objetivam definir um indicador de desempenho, o nível do Scrum, para gerir a implementação do Scrum a partir do DMAIC.

Enquanto o DMAIC é tradicionalmente implementado a partir do modelo em cascata (o progresso de uma fase para a próxima se dá de uma forma puramente sequencial), o Agile DMAIC se propõe a utilizá-lo de forma iterativa e incremental, possibilitando que a duração de seu ciclo seja reduzida para 1 a 16 semanas (1 a 4 Sprints do Scrum), potencializando a antecipação da implantação de melhorias em relação ao uso tradicional do DMAIC.

\section{Resultados}

Os projetos reais utilizados como estudo de caso deste trabalho são desenvolvidos em laboratórios de P\&D\&I do Grupo de Redes de Computadores, Engenharia de Software e Sistemas (GREat) ${ }^{1}$ da Universidade Federal do Ceará (UFC). Um resumo da caracterização dos projetos e de suas principais dificuldades é apresentado na Tabela 5. Esses projetos são voltados à Pesquisa (P), Desenvolvimento (D) e Manutenção (M) de aplicações para smartphones. Antes do Agile DMAIC, os projetos App1, App2, App3 e App7 já utilizavam o Scrum, enquanto os demais passaram a adotá-lo a partir do Agile DMAIC, com exceção do projeto App5 que se decidiu por não utilizá-lo. A inclusão do projeto App5 nesse estudo de caso é realizada para verificar que o Agile DMAIC consegue diferenciar entre os projetos que usam o Scrum e aqueles que não o utilizam.

Tabela 5. Resumo da Caracterização dos Projetos

\begin{tabular}{|c|c|c|c|c|l|}
\hline Projeto & Tipo & Duração & Equipe & Complexidade & \multicolumn{1}{|c|}{ Principais Dificuldades } \\
\hline App1 & D & 9 meses & 8 & Alta & Comunicação com o cliente \\
\hline App2 & P\&D & 9 meses & 7 & Alta & $\begin{array}{l}\text { Comunicação com cliente, complexidade da tecnologia, fre- } \\
\text { quentes mudanças de escopo }\end{array}$ \\
\hline App3 & P\&D & 9 meses & 8 & Alta & Comunicação com o cliente e complexidade da tecnologia \\
\hline App4 & D\&M & 2 anos & 6 & Média & $\begin{array}{l}\text { Comunicação com o cliente, dificuldade de concepção do pro- } \\
\text { duto, frequentes mudanças de prioridade e escopo }\end{array}$ \\
\hline App5 & P\&M & 2 anos & 26 & Alta & $\begin{array}{l}\text { Frequentes mudanças de escopo e prazo, grande dependência de } \\
\text { informações do produto, alta rotatividade da equipe }\end{array}$ \\
\hline App6 & P\&D & 6 meses & 6 & Alta & $\begin{array}{l}\text { Complexidade técnica da pesquisa e do design de interfaces } \\
\text { (grande quantidade de dados de entrada) }\end{array}$ \\
\hline App7 & P\&D & 9 meses & 10 & Alta & Comunicação com o cliente, complexidade da tecnologia \\
\hline
\end{tabular}

A adoção do Agile DMAIC foi antecedida por treinamentos teóricos e práticos aos principais envolvidos. Nesses treinamentos, os fundamentos, princípios, valores, papéis, artefatos e eventos do Scrum foram apresentados e discutidos, e algumas práticas foram realizadas para a fixação dos novos conceitos. Uma visão da linha de tempo com os marcos de início dos projetos, realização de treinamentos, adoção do Scrum e Agile DMAIC é apresentada na Figura 5. Como exemplo, o projeto App6 iniciou em Janeiro, participou do Treinamento de Scrum, adotou o Scrum e Agile DMAIC em Fevereiro e realizou suas avaliações em Fevereiro, Março e Abril de 2014.

Como exemplo do uso do Agile DMAIC, os diagramas de Causa e Efeito e Pareto do projeto App1 para o mês de Janeiro são apresentados na Figura 6. Esses diagramas foram elaborados na fase Analisar do DMAIC (passos 5, 6 e 7 da Tabela 4) a partir da coleta com o $C A S$, aplicado via Web à equipe. O diagrama de Causa e Efeito pode ser utilizado para discutir os principais problemas e causas do atual nível do Scrum com a equipe e a

${ }^{1}$ http://great.ufc.br/ 


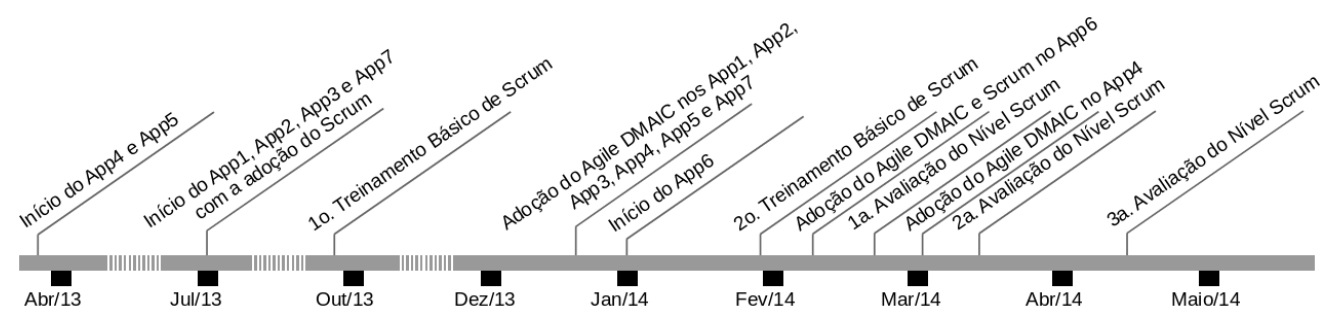

Figura 5. Linha de Tempo dos Treinamentos, da Adoção do Scrum e Agile DMAIC nos Projetos

visão dos principais ofensores, priorizados de acordo com a customização do $C A S$, pode ser obtida com o uso do diagrama de Pareto. Com esses diagramas do projeto Appl, por exemplo, é possível identificar que essa equipe deve priorizar a realização das Reuniões de Revisão, a elaboração do Plano de Lançamentos utilizando a Velocidade do time, realizar os Testes de Aceitação a cada iteração, identificar e solucionar as causas do Time está sendo interrompido e controlado de fora, bem como passar a priorizar o Product Backlog por valor de negócio.
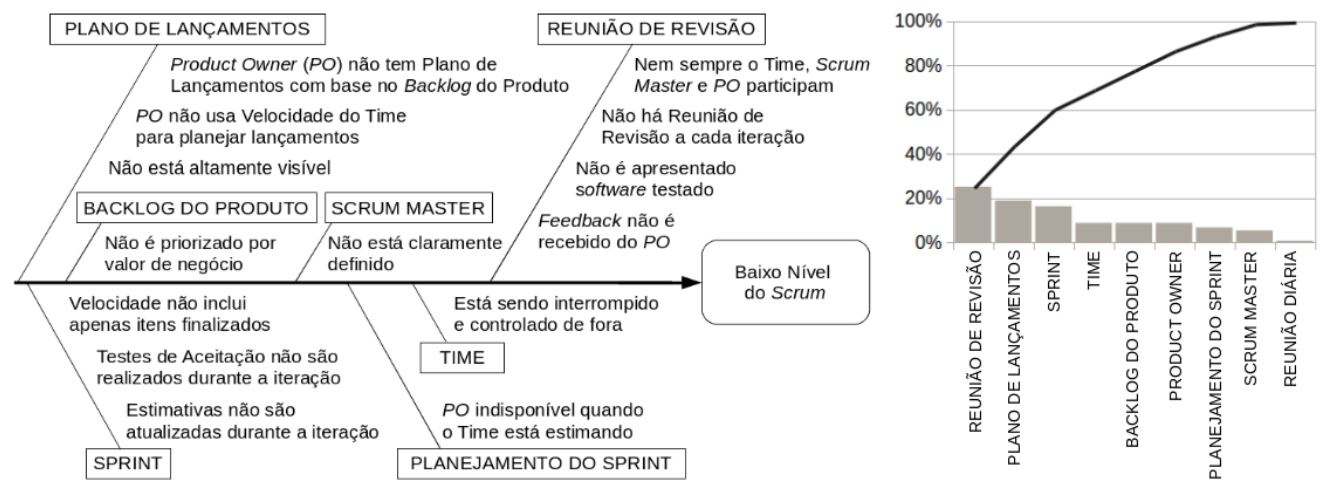

Figura 6. Diagramas de Causa e Efeito e Pareto do Projeto App1 em Janeiro/14

O gráfico com o resultado consolidado das avaliações do projeto Appl é apresentado na Figura 7. De acordo com a análise da equipe, esse projeto obteve impacto em Março por falta de prioridade do Product Owner. Embora tenham sido identificadas e implementadas ações de melhoria como a definição de um Scrum Master, a realização de Reuniões de Retrospectiva e a melhor execução de atividades de planejamento (priorização do Product Backlog, alocação e estimativas realizadas pelo time), o indicador foi impactado pela ausência desse Product Owner nas atividades do projeto.

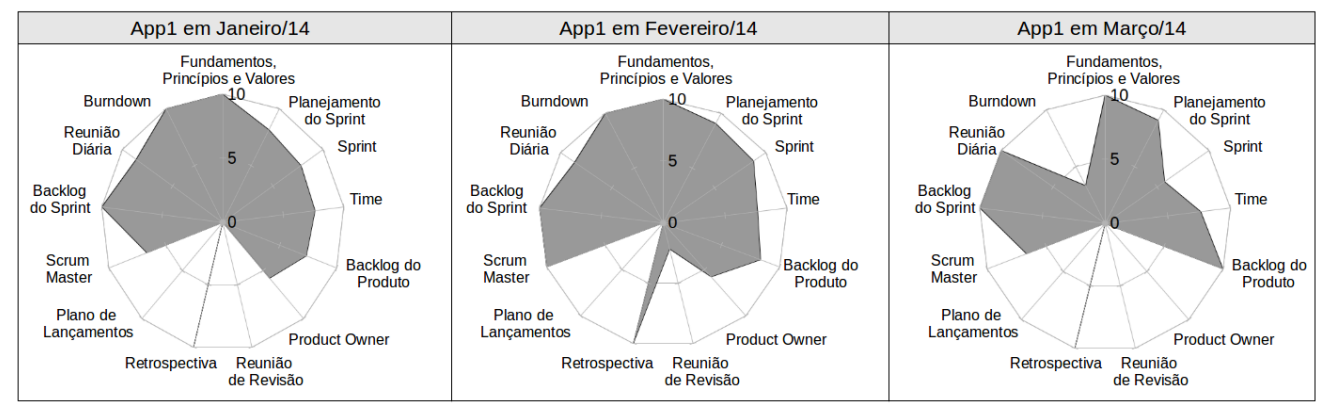

Figura 7. Resultado das Avaliações do Projeto App1 
O gráfico de Boxplot dos projetos é apresentado na Figura 8. O projeto App5 obteve o nível mais baixo por não usar o Scrum e, quanto aos demais, as análises das equipes confirmaram que o novo método refletiu a realidade do uso do Scrum nos meses avaliados. A variação desse indicador do nível nem sempre foi positiva em todos os meses. Entretanto, em análises detalhadas, os problemas identificados com o uso do método e que refletiram em uma variação negativa também foram comprovados pelas equipes. O projeto App6 obteve maior variação (positiva) a partir de ações de melhoria identificadas com o Agile DMAIC, como a necessidade de definições mais claras dos papéis (Scrum Master e Product Owner) e a resolução de falhas de comunicação (entendimento da equipe), bem como a melhoria no uso de práticas e artefatos.

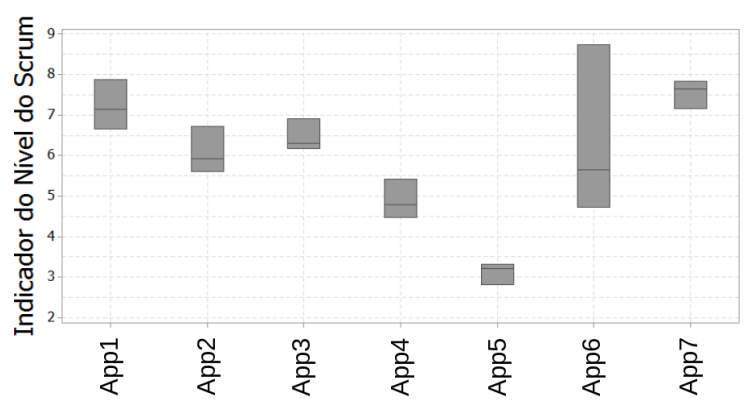

Figura 8. Boxplot dos Projetos

O gráfico de controle dos projetos que utilizam o Scrum é apresentado na Figura 9. Esses projetos apresentaram uma média de 6,4 para o indicador do nível do Scrum e ficaram abaixo da meta estabelecida ou limite de especificação inferior $(8,0)$, que implica em uma baixa capacidade atual do processo. Essa meta foi definida a partir da análise da primeira coleta dos projetos, referente a Janeiro/14, e da avaliação dos principais ofensores no uso do Scrum nesses projetos. Entretanto, mesmo nessa fase inicial, as equipes concordaram que o Agile DMAIC foi coerente em suas avaliações e que possibilitou a indentificação e resolução de problemas no uso do Scrum.

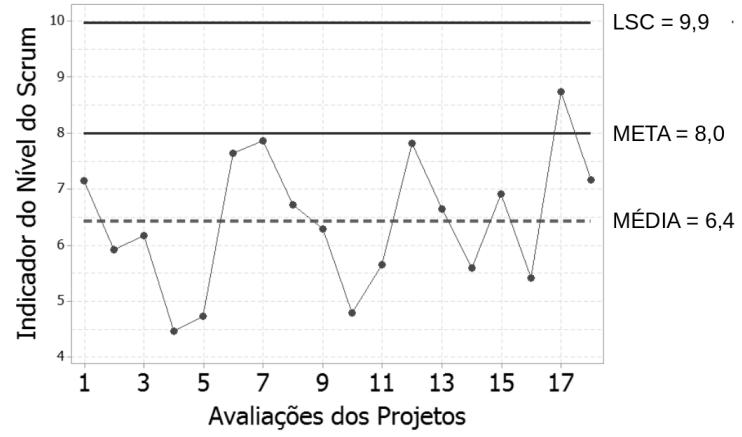

Figura 9. Controle dos Projetos que Utilizam o Scrum

Do ponto de vista dos projetos, o método contribui para a melhoria do uso dos princípios e práticas do Scrum. A partir dessa melhoria, há a expectiva que as ações já implantadas influenciem positivamente na produtividade da equipe e qualidade dos produtos [Layman et al. 2004][Dybå and Dingsøyr 2008]. Entretanto, ainda há a necessidade de mais avaliações com o Agile DMAIC nesses projetos para apresentar dados que correlacionem a evolução do nível do Scrum com a melhoria da produtividade, qualidade e satisfação do cliente. 


\section{Conclusão e Trabalhos Futuros}

Este artigo propôs o Agile DMAIC, um método para avaliação e melhoria do uso do Scrum, o qual foi aplicado em projetos reais, de complexidades média a alta, voltados à pesquisa, desenvolvimento e manutenção de software. A análise dos resultados do novo método, realizada em conjunto com as equipes, possibilitou identificar que, mesmo com certo tempo e experiência com o Scrum, essas equipes necessitavam avaliar de que forma estavam utilizando os princípios e práticas dessa metodologia.

Essa avaliação periódica de forças e fraquezas da implementação do Scrum, a partir da percepção da equipe, possibilitou identificar e tratar os principais problemas no uso dessa metodologia. Dessa forma, diante dos modelos de maturidade e checklists de avaliação de práticas ágeis analisados neste trabalho, o Agile DMAIC se mostrou uma alternativa simples, de fácil aplicação e capaz de produzir bons resultados mesmo no curto prazo.

Como trabalhos futuros, pretende-se aprimorar o Agile DMAIC a partir da simplificação de seus componentes. Os mapeamentos de níveis em práticas ágeis e de práticas em itens de seu checklist podem ser revisados. Além disso, os passos do DMAIC podem ser documentados mais detalhadamente. Uma automação do Agile DMAIC pode ser proposta a partir da implementação do checklist, mapeamentos e do DMAIC com o uso de ferramentas de workflow. Além disso, pretende-se avaliar os custos-benefícios da adoção desse novo método a médio e longo prazo, por exemplo, a relação entre o uso do mesmo e a melhoria na produtividade, qualidade e satisfação do cliente, e, por fim, verificar o seu comportamento em outros projetos, com diferentes perfis em relação aos já avaliados, bem como em outras organizações.

\section{Referências}

Balkanski, P. (2008). Team dysfunctions and scrum. Disponivel em: http: //www.scrumalliance.org/community/articles/2008/october/ team-dysfunctions-and-scrum. Acesso em: 07 Abril de 2014.

Beck (2002). Test Driven Development: By Example. Addison-Wesley Longman Publishing Co., Inc., Boston, MA, USA.

Beck, K. and Andres, C. (2004). Extreme Programming Explained: Embrace Change (2Nd Edition). Addison-Wesley Professional.

Beedle, M., Devos, M., Sharon, Y., Schwaber, K., and Sutherland, J. (2000). Scrum: An extension pattern language for hyperproductive software development.

Bezerra, C. I. M., Coelho, C. C., Gonçalves, F. M. G. S., Pires, C. G. S., and Telles, Gabriela, A. A. B. (2007). Minidmaic: Uma abordagem para análise e resolução de causas em projetos de desenvolvimento de software. VIII Simpósio Brasileiro de Qualidade de Software.

Cohn, M. (2009). Succeeding with Agile: Software Development Using Scrum. AddisonWesley Professional. ISBN 978-0321579362.

Crescêncio, S. (2013). Disfunções do scrum. Disponivel em: http://oncast.com . br/blog/ ? $\mathrm{p}=1515$. Acesso em: 07 Abril de 2014.

Cunha, T., Dantas, V., and Andrade, R. M. C. (2011). Sless: A scrum and lean six sigma integration approach for the development of sofware customization for mobile phones. In Software Engineering (SBES), 2011 25th Brazilian Symposium on, pages 283 -292. 
Dybå, T. and Dingsøyr, T. (2008). Empirical studies of agile software development: A systematic review. Inf. Softw. Technol., 50:833-859.

George, M. L. (2003). Lean Six Sigma For Service: How to Use Lean Speed and Six Sigma Quality to Improve Services and Transactions. McGraw-Hill.

Kniberg, H. (2009). Scrum checklist - version 2.0. Disponivel em: http://blog . crisp.se/2009/08/14/henrikkniberg/1250265360000. Acesso em: 14 Abril de 2014.

Layman, L., Williams, L. A., and Cunningham, L. (2004). Exploring extreme programming in context: An industrial case study. In Agile Development Conference, pages 32-41.

Moe, N. and Dingsøyr, T. (2008). Scrum and team effectiveness: Theory and practice. In Abrahamsson, P., Baskerville, R., Conboy, K., Fitzgerald, B., Morgan, L., and Wang, X., editors, Agile Processes in Software Engineering and Extreme Programming, volume 9 of Lecture Notes in Business Information Processing, pages 11-20. Springer Berlin Heidelberg.

Pan, Z., Park, H., Baik, J., and Choi, H. (2007). A six sigma framework for software process improvements and its implementation. In Software Engineering Conference, 2007. APSEC 2007. 14th Asia-Pacific, pages 446 -453.

Poppendieck, M. (2005). Principles of lean thinking.

Qumer, A., Henderson-sellers, B., and Mcbride, T. (2007). Agile adoption and improvement model.

Rubin, K. S. (2012). Essential Scrum: A Practical Guide to the Most Popular Agile Process. Addison-Wesley Professional, 1st edition.

Schwaber, K. (1995). Scrum development process. In Proceedings of the 10th Annual ACM Conference on Object Oriented Programming Systems, Languages, and Applications (OOPSLA, pages 117-134.

Schwaber, K. (2007a). Agile Project Management with Scrum. Microsoft Press.

Schwaber, K. (2007b). The Enterprise and Scrum. Microsoft Press.

Schwaber, K. and Sutherland, J. (2011). Guia do scrum, um guia definitivo para o scrum: As regras do jogo. Disponivel em: http://www.scrum.org/Portals/o/ Documents/ScrumGuides/ScrumGuide-PortugueseBR.pdf\#zoom=100. Acesso em: 23 Abril 2014.

Sidky, A., Arthur, J., and Bohner, S. (2007). A disciplined approach to adopting agile practices: the agile adoption framework. Innovations in Systems and Software Engineering, 3(3):203-216.

Tayntor, C. B. (2007). Six Sigma Software Development; 2nd ed. Taylor \& Francis Ltd, Hoboken, NJ.

Vode, B. and Sutherland, J. (2008). Scrum but test. Disponivel em: http:// antoine.vernois.net/scrumbut/?page=test\&lang=en. Acesso em: 14 Abril de 2014.

Werkema, C. (2012). CRIANDO A CULTURA LEAN SEIS SIGMA. ENGENHARIA. Elsevier. 